\title{
Transition metal precipitates in mc Si: a new detection method using 3D-FIB
}

\author{
Annika Zuschlag ${ }^{1,}$, Michail Schwab ${ }^{1}$, Dorit Merhof ${ }^{1}$ and Giso Hahn ${ }^{1}$ \\ ${ }^{1}$ University of Konstanz, 78457 Konstanz, Germany \\ aannika.zuschlag@uni-konstanz.de (corresponding author)
}

Keywords: crystalline, silicon, solar cells, transition metals, precipitates

\begin{abstract}
To investigate transition metal precipitates in $\mathrm{Si}$, synchrotron based measurements, like micro $\mathrm{x}$-ray fluorescence ( $\mu \mathrm{XRF}$ ) or detailed transmission electron microscopy (TEM) studies, are usually necessary. Transition metals are among the most detrimental defects in multi-crystalline (mc) silicon material for solar cell applications, due to their impact on minority charge carrier lifetime and possible shunt formation. We present another possibility to investigate transition metal precipitates by 3-dimensional focused ion beam (3D-FIB) cutting using a combined scanning electron microscope (SEM) SEM-FIB-system. This method is able to detect transition metal precipitates down to $5 \mathrm{~nm}$ in radius and provides additional information about the 3D shape, size and spatial distribution of the precipitates.
\end{abstract}

\section{Introduction}

The material quality of Si wafers depends on the distribution of crystallographic defects. The most obvious defects in me Si materials for solar cell applications are grain boundaries, dislocations and transition metals. The detection of transition metal precipitates in silicon materials using $\mu \mathrm{XRF}$ or TEM is well known and described e.g. in [1-6]. Electron beam induced current (EBIC) precharacterization of the samples is helpful to define regions of interest because the recombination activity of structural crystallographic defects is enhanced if those defects are decorated by transition metal impurities [7]. Falkenberg et al. [8] have demonstrated that EBIC pre-characterization provides the possibility to prepare TEM lamellas exactly at the position of highly recombination active regions and therefore allows for the successful measurement of transition metal precipitates. In this work we use the same approach and perform SEM images of a series of single FIB cuts providing data for a 3D reconstruction of the analyzed sample area. Further image processing allows to extract details of interest (here: transition metal precipitates) visible in a series of SEM images and to generate a 3D reconstruction. This so called 3D-FIB method has been firstly applied in photovoltaics for the analysis of the origin of so called "ghost plating" on the solar cell surface, using $\mathrm{Ni} / \mathrm{Cu}$ contact formation via light induced electrical plating [9].

\section{Experiment and measurement methods}

To enhance the transition metal concentration in the mc block cast silicon material, $20 \mathrm{ppma} \mathrm{Cu}$ were added to the silicon melt. This approach has the advantage that metal atoms are already present during crystallization and are not incorporated after crystallization and defect formation, which possibly results in different distributions of transition metal precipitates. Due to their small segregation coefficients, the highest concentration of these impurities is found in the top region of the $\mathrm{mc}$ Si ingot. Neutron activation analysis results in an overall concentration of approximately $1 * 10^{18} \mathrm{at} / \mathrm{cm}^{3} \mathrm{Cu}$ for the investigated samples from the top of the ingot [10].

The 3D-FIB approach is realized by using a combined SEM-FIB-system (Neon 40 EsB from Zeiss NTS GmbH), which is additionally equipped with an energy dispersive x-ray (EDX) detector and an EBIC system. The contaminated wafers were chemically polished to remove the saw damage. Further sample processing is not necessary for the following 3D-FIB investigation (except in case of EBIC pre-characterization). 
Based on EBIC pre-characterization of the $\mathrm{Cu}$ contaminated wafers, several highly recombination active defects were chosen for further 3D-FIB investigations. First of all, a 3D-FIB investigation requires the usual FIB cutting of a cross-section at the area of interest. Afterwards, single FIB cuts with a distance between 5.5-25 nm are performed. SEM images have been recorded automatically after each FIB cut and are further analyzed to extract only the transition metal precipitates within the investigated sample area.

After aligning the images for correct 3D analysis using Fast Fourier Transform by the Image Processing Extension [11] of KNIME [12], the backgrounds and sample surfaces are blanked out. Thresholds help selecting the foreground and provide 3D shapes in the image. By calculating 3D properties like number of pixels and geometric 2D properties like circularity and perimeter for each of these shapes, they can be categorized into a group of precipitates and a group of shapes being in the foreground because of image effects like the curtaining effect. The 2D properties are calculated for each $2 \mathrm{D}$ frame the 3D shape contains, and are then aggregated using various methods like geometric mean or standard deviation.

Within the $3 \mathrm{D}$ reconstruction it was defined that at least five pixels with a different contrast value regarding the surrounding silicon matrix have to be connected to be identified as a precipitate. The choosing of five pixels corresponds well to the counting of precipitates which has been performed manually for several single SEM images. Therefore, the volume of the detected precipitates is defined by the pixel size of the SEM image and the distance between two single FIB cuts. The pixel size of the SEM image is influenced by the magnification and the image resolution. In principle, the spatial resolution of the used SEM system $(1.1 \mathrm{~nm} @ 20 \mathrm{kV}, 2.5 \mathrm{~nm} @ 1 \mathrm{kV})$ can be achieved. The distance between two FIB cuts depends on the applied FIB current. The investigated volume during one $3 \mathrm{D}$-FIB cut is usually in the range of $25 \times 15 \times 15 \mu \mathrm{m}^{3}$.

Regarding all measurement parameters for the results shown in the figures below, precipitates with a radius of $7.6 \mathrm{~nm}$ or larger can be detected. Using measurement parameters allowing for a higher resolution, even smaller precipitates with $\mathrm{r}=5 \mathrm{~nm}$ can be detected, which is comparable or even below the detection limit of high resolution $\mu \mathrm{XRF}$.

\section{Results}

A comparison of the EBIC pre-characterization measurements with standard SEM images of the different regions of interest at the sample surface results in a classification of highly recombination active defects in three different groups of extended crystal defects. Fig. 1a) indicates the different groups in an overview EBIC image. The first group (A) consists of recombination active centres caused by silicon nitride inclusions within the grain. Etch pits, assorted in different lines parallel or perpendicular to each other, are visible at the sample surface around the silicon nitride needle.

The structure of these etch pit lines corresponds to an enhanced recombination activity in the EBIC measurements, as can be seen by comparison of the detailed EBIC and SEM image in Fig. 1b) and d). Similar etch pit alignments are found at the second group (B) of defects, while there is no inclusion of another material phase visible. The orientation of the etch pit lines is the same if several defected areas of type (A) or (B) are found within a grain. This hints at a relation between etch pit alignments and the crystallographic orientation of the grain, but was not further investigated. It has to be mentioned that the recombination active defects of the first two groups are located within a grain and not at a grain boundary. The etch pits are caused by a standard chemical saw damage etch and not by a special defect etch solution. Only the third group of investigated recombination active defects $(C)$ consists of grain boundaries.

In case of groups (A) and (B) a weak Cu EDX signal is measured locally at some points in the defected area. Regarding the detection limit of the EDX measurement in the SEM this hints towards an extremely high local $\mathrm{Cu}$ concentration at these defects. $\mu \mathrm{XRF}$ investigations at silicon nitride inclusions were described in [13] resulting in an enhanced transition metal content. Fig. 1c) shows a $\mu \mathrm{XRF}$ measurement of the investigated area. Due to the enhanced detection limit compared to EDX, a correlation between the recombination activity and the $\mathrm{Cu}$ contamination is visible. 

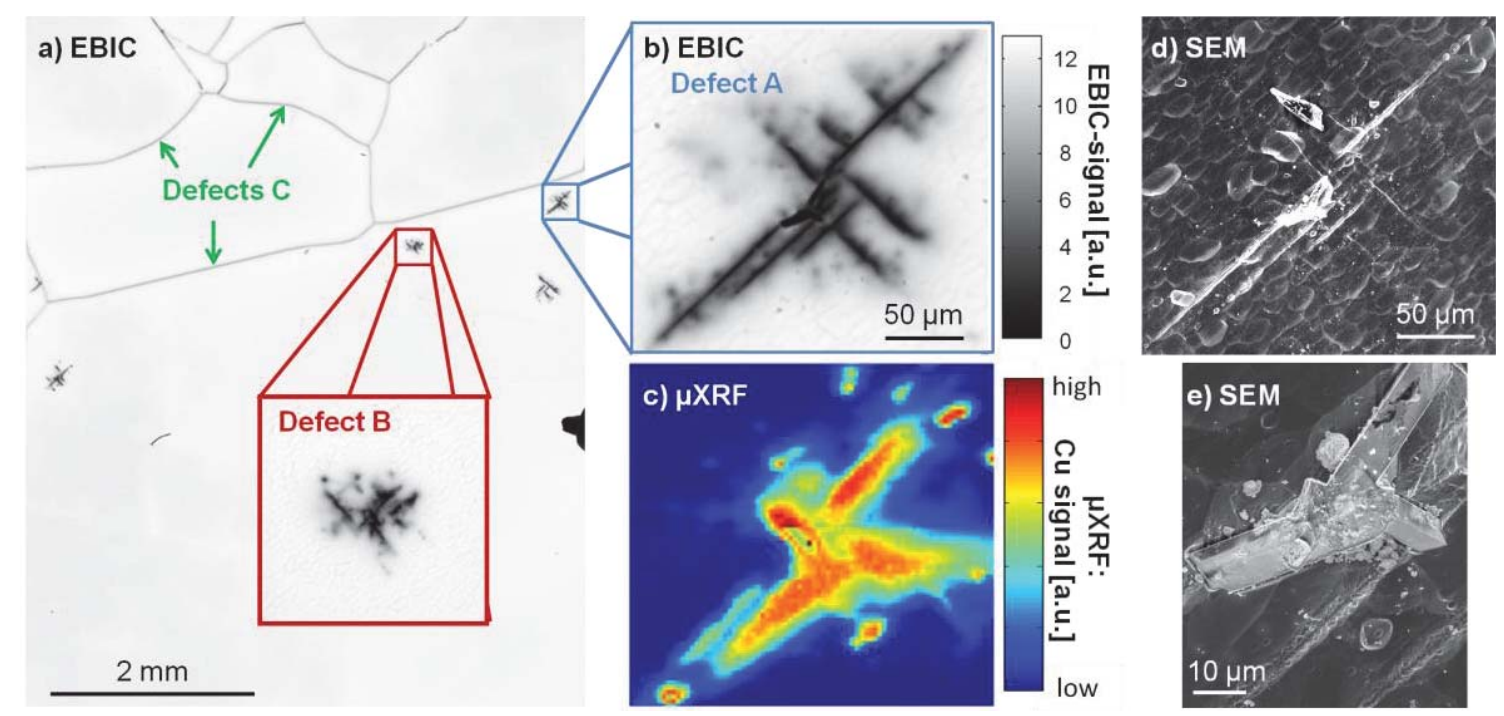

Fig. 1: a) EBIC measurement: examples for the three different defect groups investigated in this work. b) Detailed EBIC image of a group (A) defect. Lines of enhanced recombination activity are clearly visible. c) corresponding map of the $\mathrm{Cu}$ distribution measured by $\mu \mathrm{XRF}$. A strong correlation between enhanced recombination activity b) and $\mathrm{Cu}$ signal c) is found. $\mathrm{d}$ ) the sample surface shows a web of aligned etch pits by SEM investigations. The aligned etch pits correspond well to the enhanced recombination signal in b). e) detailed SEM image of the silicon nitride needle in the center of this defect structure.

Several 3D-FIB cuts were performed at these different classified defects. Usually the FIB cuts were performed perpendicular to these grain boundaries or etch pit alignments. Only in some cases additional cuts parallel to the recombination active defect were applied. All measurements on defects of group (A) and (B) have in common that a large amount of particles are detected below the etch pits. Due to the intentional contamination and the results of the previously mentioned EDX and $\mu \mathrm{XRF}$ measurement, it is assumed that these particles are $\mathrm{Cu}$ precipitates. The distribution of transition metal precipitates ends almost abruptly with the enhanced recombination activity measured by EBIC. This supports the results of the $\mu \mathrm{XRF}$ investigation concluding that the transition metals are mainly responsible for the observed recombination effects in this sample area. Further detailed results vary for the three different groups and will be discussed separately.

(A) Recombination active defects around a silicon nitride inclusion. The massive inclusion with lateral dimensions at the sample surface in the range of $70 \times 40 \mu \mathrm{m}^{2}$ in the center of this recombination active defect is identified as a silicon nitride needle by EDX measurements. The sample surface area covered by this etch pit web is approximately $200 \mathrm{x} 150 \mu \mathrm{m}^{2}$. This indicates that the silicon nitride inclusion leads to interruptions in the silicon matrix and causes further crystal defects like stacking faults, dislocations or stresses.

A sample volume of approximately $40,000 \mu \mathrm{m}^{3}$ has been investigated in several 3D-FIB cuts around the silicon nitride needle. The depth of the FIB cuts is around 15-20 $\mu \mathrm{m}$. Fig. 2 shows single SEM images detected during FIB cutting perpendicular (a) and parallel (b) to the etch pit line. In Fig. $2 b)$ the silicon nitride needle is clearly visible as well as hundreds of precipitates located only below etch pit lines and not in the surrounding sample area. All these precipitates are assorted in certain planes within the sample, as can be seen in Fig. 2a).

The density of the detected particles within the planes below the etch pits is remarkable. In Fig. $2 b$ ) up to 40 precipitates per $\mu \mathrm{m}^{2}$ were found with an average diameter of approximately $35 \mathrm{~nm}$. The smallest particles have radii of $7.6 \mathrm{~nm}$ which goes along with the detection limit of the chosen measurement parameters. This enormous assembly of precipitates hints to a highly disturbed area providing several nucleation spots for precipitates. 


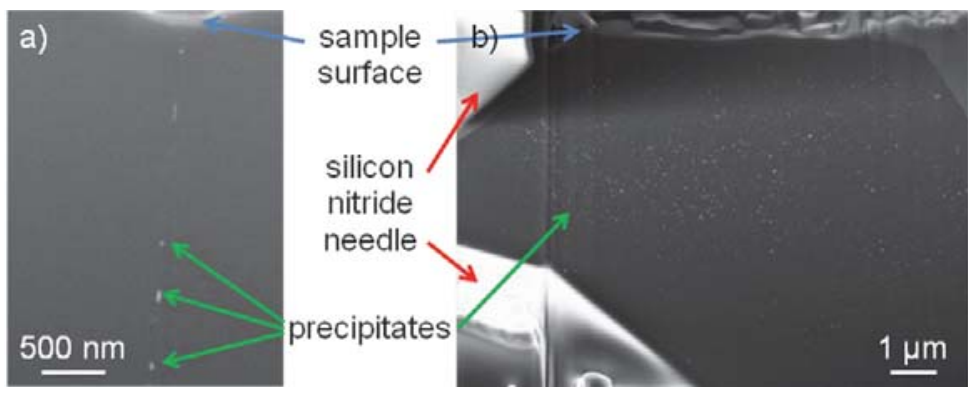

Fig. 2: Single SEM images recorded during 3D-FIB analysis perpendicular (a) or parallel to an etch pit line (b).

(B) Recombination active defects indicated by etch pit alignments. All statements of group (A) defects regarding surface structure, etch pits and recombination activity can be completely transferred to the second group of investigated regions (B). The only difference between group (A) and (B) is that in case (B) no extended inclusion in the centre of the recombination active defect structure is visible. It cannot be excluded that another silicon nitride needle is present underneath the sample surface or at the corresponding area on a directly neighbouring wafer. The observed etch pit alignments could be branches of large inclusions or other disruptions on a neighbouring wafer. Nevertheless, the 3D-FIB results show a behaviour different to case (A) and will therefore be discussed separately below.

All of the investigated areas of type (B) have in common that the precipitates are also assembled in a certain plane within the sample. Fig. 3 shows a single SEM images (a) as well as the 3D reconstruction (b) and (c) of the particle distribution of the same region from two different angles to demonstrate the perfectly aligned precipitates. Compared to defects of group (A), type (B) defects correspond to a much lower particle density, but some precipitates are much larger resulting in a size distribution from the detection limit to the range of a few microns.
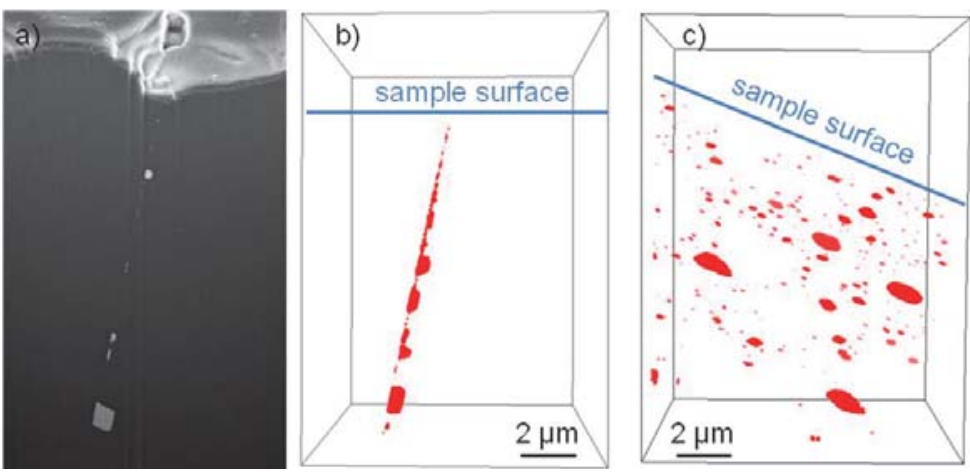

Fig. 3: SEM image a) indicates different shapes of precipitates. The $3 \mathrm{D}$ reconstructions in $\mathrm{b}$ ) and $\mathrm{c}$ ) demonstrate the distribution of the precipitates, their different sizes and shapes.

Two different shapes are observed especially for larger precipitates: a disc-like and a more or less spherical or angular shape. The disc-like precipitates lie within the plane while all precipitates with an angular shape stand out of the planes and just touch them from one and the same side as can be seen in Fig. 3b). This leads to the assumption that local structural conditions of the silicon matrix as well as stress fields strongly influence the formation of precipitates, but this was not yet further investigated in comparison to the results of the formation of precipitates in the TEM study of [14].

Detailed information about the chemical state and composition of the $\mathrm{Cu}$ precipitates cannot be given by 3D-FIB reconstruction. Only for larger precipitates with diameters of a few hundred nm it is possible to interrupt the 3D-FIB cut and analyze the chemical composition of the precipitates by EDX within the SEM-FIB-system. Fig. 4 shows a SEM image of particles (a) and the Cu signal of a corresponding EDX mapping (b). Due to the limitations of the EDX method, it is obvious that the detection of smaller precipitates is not possible. For larger precipitates the EDX Cu mapping is in good agreement with the precipitates found in the SEM image. Other materials apart from $\mathrm{Cu}$ could not be clearly detected at the precipitates.

These different observations regarding the size and distribution of transition metal precipitates compared to defect structures of type (A) suggest that another defect structure than a silicon nitride inclusion is responsible for the local crystallographic interruptions. In this case TEM measurements are needed to further analyze this sample area. 

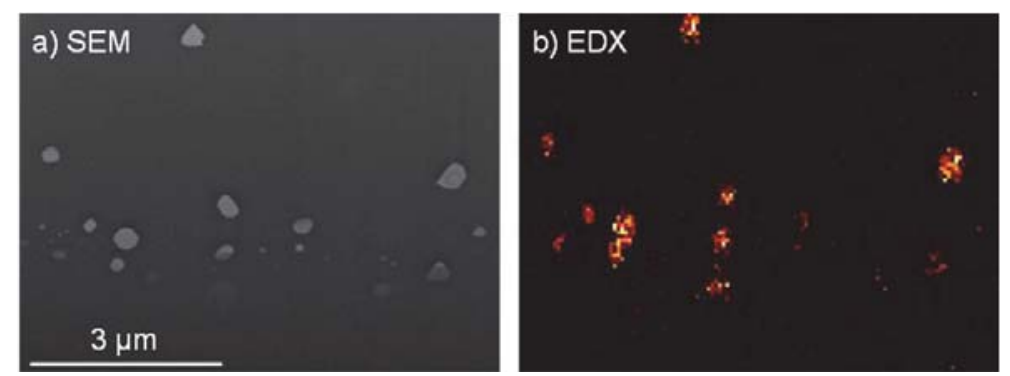

Fig. 4: a) several larger precipitates are visible in the SEM image. b) the corresponding EDX mapping of the $\mathrm{Cu}$ signal shows a strong correlation between enhanced $\mathrm{Cu}$ signal and the particles, indicating $\mathrm{Cu}$-rich precipitates.

(C) Recombination active grain boundaries. Several other measurement methods revealed that transition metal precipitates can be found at recombination active grain boundaries [15]. Three 3DFIB cuts (each approx. $25 \times 15 \times 15 \mu \mathrm{m}^{3}$ ) were performed at different highly recombination active grain boundaries, but nearly no transition metal precipitates could be detected within the investigated volumes. The used measurement parameters allow the detection of precipitates with radii larger than $15 \mathrm{~nm}$. Regarding the small investigated volumes and the limited detection limit, this 3D-FIB investigation cannot exclude the presence of transition metal precipitates at these grain boundaries. It is possible that smaller precipitates are located at the grain boundary or their density is much lower compared to the investigated areas of group (A) and (B). Also the previously mentioned other measurement methods like $\mu \mathrm{XRF}$ or TEM have shown very different transition metal precipitate distributions at recombination active grain boundaries [e.g. 16]. Similar transition metal precipitate distributions at grain boundaries compared to defects of group (B) are only found if deep etch pits along the grain boundaries indicate highly disordered areas.

\section{Discussion}

The 3D-FIB investigations show that the arrangement of precipitates depends on local conditions. The distribution, variation in size and shape vary strongly for the three different investigated areas. An interaction of transition metals and crystallographic conditions is obvious regarding the particles assembled in certain planes.

Compared to $\mu \mathrm{XRF}, 3 \mathrm{D}-\mathrm{FIB}$ is more accessible, the spatial resolution is higher and leads to additional information of the size and shape of the precipitates. The investigated sample volume of 3D-FIB is much larger than in TEM studies, but does not lead to any elemental information (except for interruption of the measurement followed by EDX analysis of larger precipitates in Fig. 4) or crystallographic information. 3D-FIB is destructive and does not allow investigation of the distribution of transition metal precipitates before and after process steps like in $\mu$ XRF studies [17].

\section{Summary}

With 3D-FIB nano-scale transition metal precipitates with radii down to $5 \mathrm{~nm}$ can be detected in $\mathrm{Si}$ wafers. 3D-FIB closes the gap of missing 3D information of precipitate distribution, size, and shape compared to $\mu \mathrm{XRF}$ and TEM studies. A combination of all three methods is necessary to obtain a detailed picture of transition metal precipitates in Si and to avoid misleading generalizations.

It could be shown that even within a sample the precipitation behaviour is strongly influenced by local crystallographic conditions. This has to be taken into account if process steps like e.g. gettering shall be optimized to reduce the transition metal content in Si material.

\section{Acknowledgement}

The authors would like to thank S. Öner for assistance with EBIC pre-characterization, and C. Dietz for assistance with image analysis. This work was supported by the Ministry of Science, Research and the Arts of Baden-Württemberg, Germany. The samples were provided by the SolarFocus project supported by the German Department of Environment and Nuclear Safety $(032765 \mathrm{H})$. 


\section{References}

[1] O.F. Vyvenko, T. Bounassisi, A.A. Istratov, E.R. Weber, M. Kittler, W. Seifert, Application of synchrotron-radiation-based x-ray microprobe techniques for the analysis of recombination activity of metal precipitated at Si/SiGe misfit dislocations, J. Phys.: Condens. Matter 14 (2002) 13079-13086

[2] T. Buonassisi, A.A. Istratov, M.D. Picket, M. Heuer, J.P. Kalejs, G. Hahn, M.A. Marcus, B. Lai, Z. Cai, S.M. Heald, T.F. Ciszek, R.F. Clark, D.W. Cunningham, A.M. Gabor, R. Jonczyk, S. Narayanan, E. Sauar, E.R. Weber, Chemical natures and distributions of metal impurities in multicrystalline silicon materials, Prog. Photovolt: Res. Appl. 14 (2006) 513-531

[3] M. Seibt, H. Hedemann, A.A. Istratov, F. Riedel, A. Sattler, W. Schröter, Structural and electrical properties of metal silicide precipitates in silicon, phys. stat. sol. (a) 171 (1999) 301 310

[4] M. Seibt, P. Saring, L. Stolze, M.A. Falkenberg, C. Rudolf, D. Abdelbarey, H. Schuhmann, Transmission electron microscopy investigations of metal-impurity-related defects in crystalline silicon, Solid State Phenom. 178-179 (2011) 275-284

[5] T. Bounassisi, A.A. Istratov, M.D. Pickett, M.A. Marcus, G. Hahn, S. Riepe, J. Isenberg, W. Warta, G. Willeke, T.F. Ciszek, E.R. Weber, Quantifying the effect of metal-rich precipitates on minority carrier diffusion length in multicrystalline silicon using synchrotronbased spectrally resolved x-ray beam-induced current, Appl. Phys. Lett. 87(1) (2005), 1-3

[6] T. Bounassisi, O. Vyvenko, A.A. Istratov, E.R. Weber, G. Hahn, D. Sontag, J.P. Rakotoniaina, O. Breitenstein, J. Isenberg, R. Schindler, Observation of transition metals at shunt locations in multicrystalline silicon solar cells, J. Appl. Phys. 95(3) (2004) 1556-1561

[7] V. Kveder, M. Kittler, W. Schröter, Recombination activity of contaminated dislocations in silicon: a model describing electron-beam-induced current contrast behavior, Phys. Rev. B 63 (2001) 115208

[8] M.A. Falkenberg, H. Schuhmann, M. Seibt, V. Radisch, Localization and preparation of recombination-active extended defects for transmission electron microscopy analysis, Rev. Sci. Instrum. 81 (2001) 063705

[9] S. Braun, A. Zuschlag, B. Raabe, G. Hahn, The origin of background plating, Energy Procedia 8 (2011) 565-570

[10] I.E. Reis, S. Riepe, W. Koch, J. Bauer, S. Beljakowa, O. Breitenstein, H. Habenicht, D. Kreßner-Kiel, G. Pensl, J. Schön, W. Seifert, Effect of impurities on solar cell parameters in intentionally contaminated multicrystalline silicon, Proc. $24^{\text {th }}$ EU PVSEC Hamburg (2009), 2144-2148

[11] http://tech.knime.org/community/image-processing

[12] http://knime.org

[13] M. Holla, T. Arguirov, W. Seifert, M. Kittler, Analysis of silicon carbide and silicon nitride precipitates in block cast multicrystalline silicon, Solid State Phenom. 156-158 (2010) 41-48

[14] M. Seibt, M. Griess, A.A. Istratov, H. Hedemann, A. Sattler, W. Schröter, Formation and properties of copper silicide precipitates in silicon, phys. stat. sol (a) 166 (1998) 171-182

[15] T. Buoanssisi, A.A. Istratov, M.D. Pickett, M.A. Marcus, T.F. Ciszek, E.R. Weber, Metal precipitation at grain boundaries in silicon: dependence on grain boundary character and dislocation decoration, Appl. Phys. Lett. 89 (2006) 042102

[16] A. Zuschlag, S. Ohl, J. Bernhard, H. Morhenn, J. Ebser, J. Junge, S. Seren, G. Hahn, $\mu$ XRF investigations of the influence of solar cell processing steps on iron and copper precipitates in multicrystalline silicon, Proc. $35^{\text {th }}$ IEEE PVSC Honolulu (2010) 347-351

[17] D.P. Fenning, A.S. Zuschlag, M.I. Bertoni, B. Lai, G. Hahn, T. Buonassisi, Improved iron gettering of contaminated multicrystalline silicon by high-temperature phosphorus diffusion, J. Appl. Phys. 113 (2013) 214504 